\title{
A New Perspective on Laboratory Proficiency Testing
}

\author{
Perez Quartey* \\ *Faculty of Heath Sciences, Garden City University College, Ghana \\ *Corresponding author: Perez Quartey, Faculty of Heath Sciences, Garden City University College, Kenyase, Ghana. \\ To Cite This Article: Perez Quartey, A New Perspective on Laboratory Proficiency Testing. 2020 - 8(1). AJBSR.MS.ID.001235. \\ DOI: 10.34297/AJBSR.2020.08.001235.
}

Received: 渄 February 18, 2020; Published: 畊 March 12, 2020

\section{Introduction}

Accreditation bodies and Regulatory agencies require the participation of clinical laboratories in periodic External Quality Assurance (EQA) programs as a means of providing an objective evidence of the quality of testing of patients' specimens [1,2]. The overall purpose of EQA is for identification of shortfalls in the total testing system of the laboratories for improvement and maintenance of quality. In addition to elucidating competencies of testing personnel, participation in periodic inter-laboratory comparability and proficiency testing programs may reveal gradual or sudden changes in performance of the testing system that a laboratory may be unaware of. Participation in an inter-laboratory comparability and proficiency testing programs can therefore help to verify the reliability and quality of testing as a means of improvement and maintenance of quality assurance.

\section{Presentation}

Proficiency testing typically involves an accredited scheme provider preparing reference materials and sending them to the participating laboratories for measurements $[3,4]$. The results from the participating labs are then returned to the scheme provider for analysis and feedback is subsequently reported to the participating labs. The whole process of conventional proficiency testing is done with the conscious awareness of the participating labs of their involvement in an EQA exercise. External quality assurance programs like proficiency testing and inter-laboratory comparison provide substantial information to measure overall testing performance. However, they do not necessarily give an indication of the daily accuracy, reproducibility and overall reliability of individual testing. EQA surveys may merely provide a snapshot in time and therefore should not be used as a substitute for daily quality.

\section{Hypothesis}

When EQA is conducted with the conscious awareness of the participating labs that the testing is being carried out as part of a formal EQA program, there is a strong likelihood of bias in adhering to strict internal quality control standards with the sole aim of achieving good results. "Acceptable" performance in an EQA testing may therefore not reflect or guarantee testing reliability on individual samples run as real daily work. A recently published article by this author, Quartey et al. [5] have raised a new perspective on the performance of inter-laboratory comparability of testing. In the study, all samples were sent to the selected labs as "blindedpatient samples" labelled with anonymous patients' names, ages, gender and attached with corresponding request forms with different clinical diagnoses. Additionally, the laboratories were not aware of any inter-laboratory comparability exercise and all results were collected as "True patient" results as routinely dispatched by the labs. All these showed that the results gavin were the most likely test reporting that would have been produced by the labs as part of their routine daily runs. The study presented the likely events of the "real-life" daily results presented to patients and requesting physicians alike when samples are handled as routine patient samples for laboratory analysis and not as samples for an external quality assurance work. The results of the study showed that many of the laboratories did not meet total analytical error goals by both European and CLIA recommendations.

\section{Conclusion}

Though clinical laboratories should be aware of periodic Proficiency Testing, accredited scheme providers would get a better view of analytical performance of the participating laboratories by employing the methodology utilized by Quartey et al. [5] as a means of improving overall quality assurance of analytical performance of laboratories.

\section{References}

1. Sciacovelli L, Secchiero S, Zardo L, Plebani M (2010) The role of the External Quality Assessment. Biochemia Medica 20(2): 160-164.

2. Kettelhut MM, Chiodini PL, Edwards H, Moody A (2003) External quality assessment programmes raise standards: evidence from the UKNEQAS parasitology subschemes. J Clin Pathol 56(12): 927-932. 
3. (2010) Conformity assessment- General requirements for proficiency testing. International Organization for Standardization Geneva.

4. (2013) Medical Laboratories-Requirements for quality and competence. International Organization for Standardization.
5. Quartey P, Anokye R, Frimpong M (2019) Inter-Laboratory Comparability of Clinical Chemistry Testing: A New Perspective. International Journal of Sciences: Basic and Applied Research 48(5): 196-207. 